\title{
COMPOSICIÓN DEL ACEITE ESENCIAL DE Tagetes parryi A. GRAY
}

\author{
COMPOSITION OF Tagetes parryi A. GRAY ESSENTIAL OIL
}

\author{
Francisco Díaz-Cedillo ${ }^{1}$ y Miguel A. Serrato-Cruz ${ }^{2 *}$
}

\begin{abstract}
${ }^{1}$ Departamento de Química Orgánica, Escuela Nacional de Ciencias Biológicas, Instituto Politécnico Nacional. Prolongación de Carpio y Plan de Ayala s/n, Casco de Santo Tomás. 11340, México, D.F. ${ }^{2}$ Departamento de Fitotecnia, Universidad Autónoma Chapingo. Km 37.5 Carr. México-Texcoco. 56250, Chapingo, Edo. de México.

${ }^{*}$ Autor para correspondencia (serratocruz@gmail.com)
\end{abstract}

\section{RESUMEN}

Es escasa la información sobre los compuestos químicos presentes en el aceite esencial de las especies mexicanas de Tagetes. Mediante hidrodestilación se extrajo aceite esencial de las partes aéreas de plantas de Tagetes parryi A. Gray (Asteraceae), planta endémica en el Estado de San Luis Potosí, México. Mediante análisis por cromatografía de gases acoplada a espectrometría de masas, se identificaron siete compuestos principales en el aceite esencial con la siguiente abundancia relativa: canfeno (96\%), 3, 6, 6-trimetil2-norpinanol (91\%), anisol (60\%), 4-isopropil-1-metil-2-ciclohexenol (50 $\%)$, cineol (48\%), eugenol (14\%) y a-terpineol (12\%). El segundo y cuarto compuestos no se habían descrito para Tagetes.

Palabras clave: Tagetes parryi, Asteraceae, canfeno, cineol.

\section{SUMMARY}

Information about chemical compounds in the essential oil for Mexican species of Tagetes is scarce. The essential oil from aerial plant parts of Tagetes parryi A. Gray (Asteraceae), an endemic plant from the State of San Luis Potosí, México, was obtained by hydrodistillation. By GC-MS analysis seven major compounds were identified in this essential oil, with the following abundance: camphene $(96 \%), 3,6,6$-trimethyl-2-norpinanole $(91 \%)$, anisole (60\%), 4-isoprophyl-1-methyl-2-cyclohexenole (50\%), cineole (48\%), eugenole (14\%). and $\alpha$-terpineole (12\%). The second and fourth chemical compounds have not been reported for Tagetes.

Index words: Tagetes parryi, Asteraceae, camphene, cineole.

\section{INTRODUCCIÓN}

Diversas publicaciones sobre el género Tagetes (Asterales: Asteraceae) (Neher, Com. pers. ${ }^{3}$; Soule, Com. pers. ${ }^{4}$ Panero y Villaseñor, 1996; Turner, 1996) permiten estimar que México cuenta con 35 especies, la mayoría de ellas aromáticas. $\mathrm{El}$ aroma en Tagetes se atribuye al tipo de metabolitos presentes en el aceite esencial localizado en estructuras especiales en toda la planta (Russin et al., 1988; Poli et al., 1995;

\footnotetext{
${ }^{3}$ Neher R T (1965) Monograph of the genus Tagetes (Compositae). Ph. D. Thesis Indiana University, USA. 306 p.

${ }^{4}$ Soule J A (1993) Systematics of Tagetes (Asteraceae-Tageteae). Doctoral Thesis. The University of Texas, Austin. USA. 683 p.
}

López et al., 2008) y en forma abundante en la parte aérea. En algunas especies mexicanas de este género se han identificado metabolitos secundarios, como en T. coronopifolia (Perez-Amador et al., 1994), T. erecta (Sefidkon et al., 2004; Ogunwade y Olawore, 2006), T. filifolia (Serrato-Cruz et al., 2008), T. lucida (Bichi et al., 1997; Cicció, 2004) y T. patula (Romagnoli et al., 2005; Sagar et al., 2005), pero aún se desconoce la composición de la mayoría de especies.

Al comparar la composición del aceite esencial de varias especies de Tagetes se concluye que algunos metabolitos son diferentes y otros son comunes (Lawrence, 1985; Héthélyi et al., 1986; Marotti et al., 2004). El aceite de Tagetes tiene actividad biológica sobre varios organismos patógenos de plantas y animales (Eguaras et al., 2005), así como en humanos (Cestari et al., 2004), por lo que este género representa una opción en agricultura y medicina como insumo natural con efecto biocida, biorrepelente o biostático.

Tagetes parryi A. Gray es una hierba o arbusto de 30 a 50 $\mathrm{cm}$ de altura que habita en forma endémica en unos pocos sitios localizados entre las zonas semiáridas y la de transición climática, al sureste del Estado de San Luis Potosí, México (Neher, 1965) ${ }^{3}$. No se cuenta con información sobre esta especie, aunque se ha propuesto para utilizarse en horticultura ornamental (Soule, 1996) ${ }^{4}$.

Recientemente, en México se han efectuado investigaciones sobre conservación, caracterización y aprovechamiento de las especies mexicanas de Tagetes distribuidas en diferentes regiones del país, especialmente para el desarrollo de bioplaguicidas (Serrato-Cruz et al., 2008). En virtud de los escasos estudios acerca de la composición del aceite esencial de T. parryi que produce un intenso aroma principalmente en su parte aérea (Turner, 1996), el objetivo del presente trabajo fue determinar la composición química del 
aceite esencial de T. parryi con la finalidad de destacar su posible uso potencial como repelente o disuasivo de plagas.

\section{MATERIALES Y MÉTODOS}

\section{Material vegetal}

En octubre de 2009 se recolectaron muestras aleatorias de la parte aérea de plantas en floración de T. parryi (Figura 1) en la Sierra de Álvarez, al sureste de la ciudad de San Luis Potosí. Información de la identificación taxonómica y especímenes de T. parryi está resguardada en el Herbario del Instituto de Investigaciones en Zonas Áridas, de la Universidad Autónoma de San Luis Potosí.

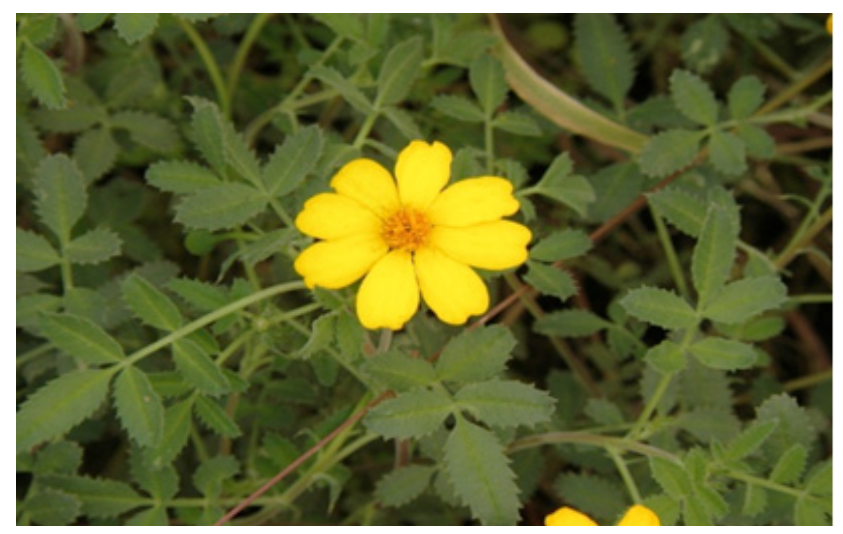

Figura 1. Tagetes parryi A. Gray en floración (Fotografía: Miguel Ángel Serrato Cruz).

\section{Obtención del aceite esencial}

Una muestra combinada de hojas, tallos e inflorescencias frescas de seis plantas en plena floración, se sometieron a condiciones de hidrodestilación por $45 \mathrm{~min}$ (Serrato-Cruz et al., 2008). Los aceites fueron secados con sulfato de sodio anhidro y almacenados a $4{ }^{\circ} \mathrm{C}$ en oscuridad.

\section{Análisis del aceite}

La composición química de los aceites esenciales se analizó por cromatografía de gases-espectrometría de masas (CG-EM) en un equipo Polaris Q Finnnigan Trace GC Ultra $\otimes$ provisto con un detector de masas Polaris Q, y con impacto electrónico de $70 \mathrm{eV}$. Se empleó una columna RTX5MX, difenil-dimetilpolisiloxano (5:95), de $30 \mathrm{~m}$ x $0.25 \mathrm{~mm}$ x $0.25 \mu \mathrm{m}$. El inyector y el detector se establecieron a 250 y $300{ }^{\circ} \mathrm{C}$. La temperatura del horno se inició en $70^{\circ} \mathrm{C}$, se mantuvo así $1 \mathrm{~min}$ y se programó para alcanzar $250^{\circ} \mathrm{C}$ a una tasa de $20^{\circ} / \mathrm{min}$. Como gas acarreador se usó helio, a

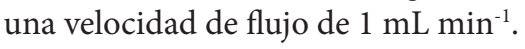

Se inyectaron manualmente muestras diluidas (1/100 en cloruro de metileno, v/v) de $1 \mu \mathrm{L}$, en modo "split" (disposi- tivo para diluir). Los datos cuantitativos de abundancia relativa se obtuvieron electrónicamente, y fueron observables en el cromatograma. El intervalo de masas detectadas fue de 35 a $500 \mathrm{~m} / \mathrm{z}$. Se usaron $n$-alcanos como referencias en el cálculo de los índices de Kovats. Se midieron tres muestras procesadas en las que la identificación de los componentes se hizo mediante comparación de los índices de retención relativa y los espectros de masas con la base de datos NIST del sistema GC-MS y con los datos de Adams publicados por Corp. Carol Stream, USA (Adams, 2001); también se hizo co-inyección con compuestos de referencia.

\section{RESULTADOS Y DISCUSIÓN}

En este primer estudio sobre la composición química del aceite esencial de T. parryi, se detectaron siete compuestos en mayor proporción que, de acuerdo con su patrón de fragmentación, aparecieron con tiempos de retención en la secuencia siguiente: 4-isopropil-1-metil-2-ciclohexenol (3.9 $\mathrm{min}$ ) , 3, 6, 6-trimetil-2-norpinanol (4.0 $\mathrm{min}$ ), cineol (4.9 $\mathrm{min})$, anisol (5.6 min), canfeno (6.7 $\mathrm{min})$, $\alpha$-terpineol (7.5 min) y eugenol (11.3 min) (Figura 2, Cuadro 1). Por su abundancia relativa, los compuestos se ubicaron en tres gradientes: 91 a $96 \%$ (canfeno, 3,6,6-trimetil-2-norpinanol), 48 a $60 \%$ (cineol, 4-isopropil-1-metil-2-ciclohexenol y anisol), y 12 a $14 \%$ (a-terpineol y eugenol) (Cuadro 1).

Cuadro 1. Componentes químicos del aceite esencial de T. parryi y sus tiempos de retención determinados mediante el análisis cromatográfico.

\begin{tabular}{lcc}
\hline Compuesto & $\begin{array}{c}\text { Abundancia } \\
\text { relativa } \\
(\%)\end{array}$ & $\begin{array}{c}\text { Tiempo de } \\
\text { retención } \\
(\mathrm{min})\end{array}$ \\
\hline Canfeno & 96 & 6.76 \\
3,6,6-trimetil-2-norpinanol & 91 & 4.05 \\
Anisol & 60 & 5.66 \\
4-isopropil-1-metil-2-ciclohexenol & 50 & 3.91 \\
Cineol & 48 & 4.97 \\
Eugenol & 14 & 11.39 \\
a- terpineol & 12 & 7.58 \\
\hline
\end{tabular}

Canfeno resultó un compuesto que no se había citado como abundante (96\%) en Tagetes; en T. minuta, por ejemplo, se presenta como un componente traza $(0.1 \%)$ (Senatore et al., 2004). Este metabolito constituye uno de los principales ingredientes activos del aceite de Acorus calamus L., que se utiliza como biocida contra larvas del mosco Aedes aegypti que transmite el dengue, y también tiene fuerte efecto biológico contra hongos y nemátodos (Tariq et al., 2010).

No se encontraron antecedentes sobre los efectos biológicos de 3,6,6-trimetil-2-norpinanol y de 4-isopropil-1- 


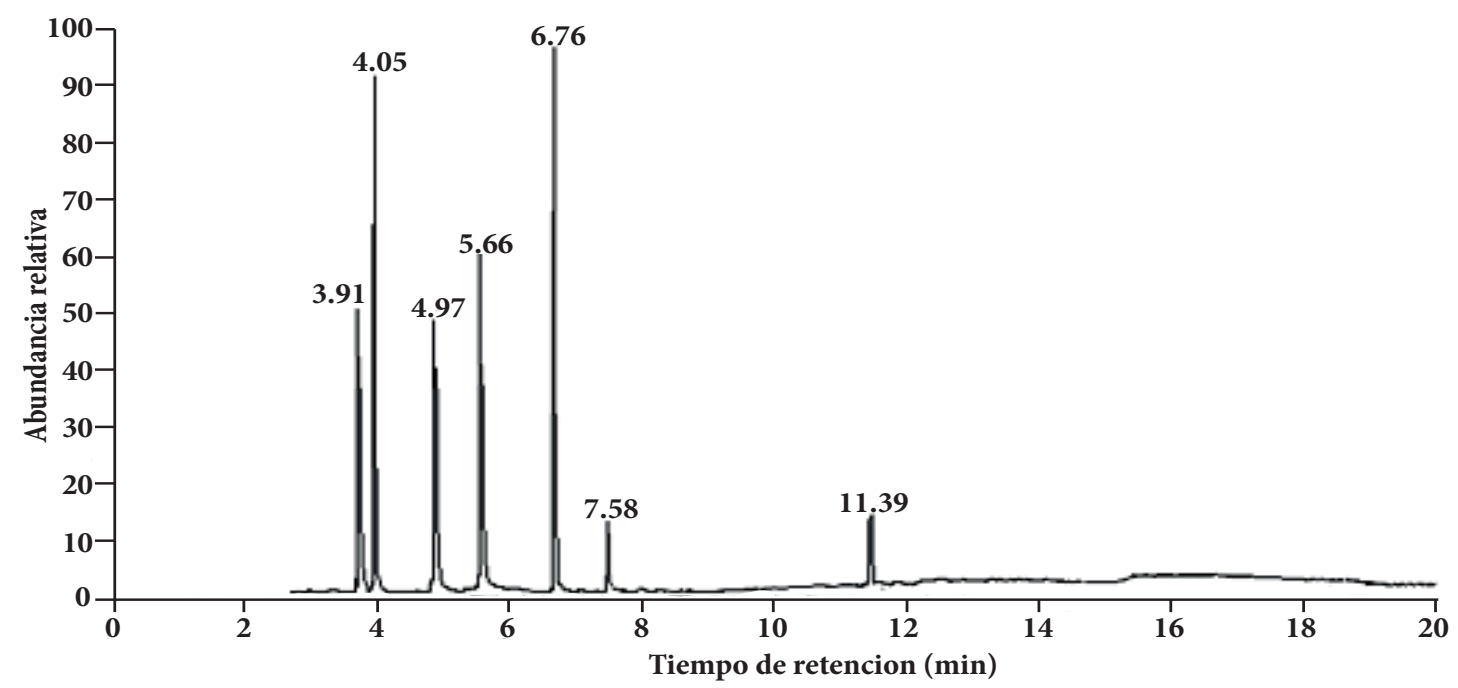

Figura 2. Cromatograma que muestra los picos de: 4-isopropil-1-metil-2-ciclohexenol (tiempo de retención, Rt, 3.9), 3,6,6-trimetil-2-norpinanol (Rt, 4.0), cineol (Rt, 4.9), anisol (Rt, 5.6), canfeno (Rt, 6.7), a-terpineol (Rt, 7.5) y eugenol (Rt, 11.3).

metil-2-ciclohexenol, que tampoco han sido descritos en el género Tagetes. Anisol es un compuesto presente en varias especies de Tagetes (Cicció, 2004; Serrato-Cruz et al., 2008) con efecto biológico contra mosquita blanca (Trialeurodes vaporariorum West) (Camarillo-de la Rosa et al., 2009). Cineol, a-terpineol y eugenol son compuestos que previamente se habían detectado en Tagetes (Marotti et al., 2004; Ogunwande y Olawore, 2006; Moghaddam et al., 2007). Se ha reportado que aceites esenciales que contienen eugenol, a-terpineol y cineol poseen actividad contra microorganismos e insectos (Araujo et al., 2003; Lucia et al., 2007), y que a-terpineol inhibe la proliferación de células cancerosas en humanos (Lambpronti et al., 2006).

Los aceites esenciales son utilizados por las plantas básicamente para su adaptación al medio, como defensa contra enemigos naturales (Wink, 1988) y para el ajuste fisiológico al ambiente (Lincoln y Langenheim, 1979), entre otras funciones. Se puede inferir entonces que probablemente los compuestos mayoritarios de T. parryi realizan esas funciones en el ambiente semiárido en el que principalmente se desarrolla esta especie (Turner, 1996). Al considerar la presencia de los siete compuestos mayoritarios en el aceite esencial de T. parryi y los antecedentes señalados sobre los efectos biológicos que ocasionan tales metabolitos secundarios encontradas en otras especies de Tagetes y en otros géneros de plantas, se puede anticipar su potencial aplicación en agricultura como bioplaguicida, e incluso su uso en medicina. Concomitantemente, el conocimiento fitoquímico de T. parryi confirma la importancia de conocer la biodiversidad de México, además de aportar información sobre tópicos especiales como la quimiotaxonomia, tan necesaria para el debate sobre la clasificación taxonómica de Tagetes.

Actualmente se están efectuando investigaciones sobre la distribución de estos compuestos en los órganos de la planta de T. parryi, ya que en otras especies de Tagetes se ha encontrado que los componentes varían estructuralmente y en su concentración en el aceite esencial, en función del órgano vegetal (Sefidkon et al., 2004).

Los aceites esenciales presentes en T. parryi y en otras especies de Tagetes son de importancia como productos de alto impacto en la agricultura ecológica (López-Olguín et al., 2007), por lo que sería conveniente investigar las propiedades toxicológicas y de repelencia que podría tener el aceite esencial de T. parryi contra las plagas.

\section{CONCLUSIONES}

En el aceite esencial de T. parryi se encontraron siete compuestos mayoritarios: canfeno, 3, 6, 6-trimetil-2-norpinanol, anisol, 4-isopropil-1-metil-2-ciclohexenol, cineol, eugenol y a-terpineol; el segundo y cuarto compuestos no se habían identificado para el género Tagetes.

\section{AGRADECIMIENTOS}

Esta investigación es una parte del proyecto "Determinación del rendimiento de aceites esenciales, composición química y características fisicoquímicas de especies aromáticas de Tagetes", el cual corresponde a la Red Cempoalxóchitl financiada por el Sistema Nacional de Recursos Fitogenéticos (SINAREFI) coordinada por el Sistema Nacional 
de Inspección y Certificación de Semillas, a través del Programa SINAREFI de la SAGARPA, México.

\section{BIBLIOGRAFÍA}

Adams R P (2001) Identification of Essential Oils Components by Gas Chromatography/Quadrupole. Mass Spectroscopy. Allured Publishing Corp, Carol Stream, IL. pp:468-572.

Araujo E C, E R Silveira, M A Lima, M A Neto, I L de Andrade, M A Lima (2003) Insecticidal activity and chemical composition of volatile oils from Hyptis martisii Benth. J. Agric. Food Chem. 51:32603262.

Bicchi C, M Fresia, P Rubiolo, D Monti, C Franz, I Goehler (1997) Constituents of Tagetes lucida Cav. ssp. lucida essential oil. Flavour Frag. J. 12:47-52.

Camarillo-de la Rosa G, L D Ortega-Arenas, Miguel A Serrato-Cruz, C Rodríguez-Hernández (2009) Actividad biológica de Tagetes filifolia Lag. (Asteraceae) en moscas blancas Trialeurodes vaporariorum Westwood (Hemíptera: Aleyroidae). Rev. Colomb. Entomol. 35:177184.

Cestari I M, S J Sarti, C M Waib, A Castello (2004) Evaluation of the potential insecticide activity of Tagetes minuta (Asteraceae) essential oil against the head lice Peduncularis humanus capilis (Phthiraptrea; Pediculidae). Neotrop. Entomol. 33:805-807.

Cicció J F (2004) A source of almost pure methyl chavicol: volatile oil from the aerial parts of Tagetes lucida (Asteraceae) cultivated in Costa Rica. Rev. Biol. Trop. 52:853-857.

Eguaras M J, S Fuselli, L Gende, R Fritz, S R Ruffinengo, G Clemente, G González, P N Bailac, M I Ponzi (2005) An in vitro evaluation of Tagetes minuta essential oil for the control of the honeybee pathogens Paeniibacillos larvae and Ascosphaera apis, and the parasitic mite Varroa destructor. J. Essential Oil Res. 17:336-340.

Héthélyi É, B Dános, P Tétényi, I Koczka (1986) GC/MS analysis of the essential oils of some Tagetes species. In: Progress in Essential Oil Research. E S Brunke (ed). Walter de Gruyter \& Co. Berlin, Germany. pp:131-137.

Lambpronti I, A M Saab, R Gambari (2006) Antiproliferative activity of essential oils derived from plants belonging to the Magnoliophyta division. Interntl. J. Oncol. 29:989-995.

Lawrence B M (1985) Essential oils of the Tagetes genus. Perfume \& Flavour 10:73-82.

Lincoln D E, Langenheim J H (1979) Variation of Satureja douglasii monoterpenoids in relation to light intensity and herbivory. Biochem. System. Ecol. 7:289-298.

López, M L, N E Bonzani, J A Zygadlo (2008) Allelopathic potential of Tagetes minuta terpenes by a chemical, anatomical and phytotoxic approach. Biochem. System. Ecol. 36:882-890.

López-Olguín J F, A Aragón-García, C Rodríguez-Hernández, M VázquezGarcía (2007) Substancias Naturales contra Plagas. Agric. Sostenible 3: 201
Lucia A, P González, E Saccacini, S Licastro, E Zerba, H Masuh (2007) Larvicidal effect of Eucalyptus grandis essential oil and turpentine and their major components on Aedes. aegypti larvae. J. Amer. Mosquito Control Assoc. 23:293-303.

Marotti M, R Piccaglia, B Biavati, I Marotti (2004) Characterization and yield evaluation of essential oils from different Tagetes species. J. Essential Oil Res. 16:440-444.

Moghaddam M, R Omidbiagi, F Sefidkon (2007) Chemical composition of the essential oil of Tagetes minuta L. J. Essential Oil Res. 19:3-4.

Ogunwande I A, N O Olawore (2006) Essential oil from the leaves and flowers of "African Marigold", Tagetes erecta L. J. Essential Oil Res. 18:366-368.

Panero J L, J L Villaseñor (1996) Novelties in Asteraceae from southern Mexico. Brittonia 48:79-90.

Pérez-Amador M C, F García-Jiménez, J Herrera, L P González, L C Márquez-Alonso (1994) Essential oils, anthocyanins and phototoxic compounds in two species of Tagetes (Asteraceae). Phyton 56:143146.

Poli F, G Sacchetti, A Bruni (1995) Distribution of internal secretory structures in Tagetes patula (Asteraceae). Nordic J. Bot. 15:197-205.

Romagnoli C, R Bruni, E Andreotti, M K Rai, C B Vicentini, D Mares (2005) Chemical characterization and antifungal activity of essential oil of capitula from wild Indian Tagetes patula L. Protoplasma 225:5765.

Russin, W A, T F Uchytil, G Feistner, R D Durbin (1988) Developmental changes in content of foliar secretory cavities of Tagetes erecta (Asteraceae). Amer. J. Bot. 75:1787-1793.

Sagar D V, S N Naik, P K Rout, Y R Rao (2005) Composition of essential oils of Tagetes patula L. growing in Northern India. J. Essential Oil Res. 17:446-448.

Sefidkon F, S Salehyar, M Mirza, M Daviri (2004) The essential oil of Tagetes erecta L. occurring in Iran. Flavour Percep. J. 19:579-581.

Senatore F, F Napolitano, M A-H Mohamed, P J C Harris, P N S Mnkeni, J Henderson (2004) Antibacterial activity of Tagetes minuta L. (Asteraceae) essential oil with different chemical composition. Flavour Frag. J. 19:574-578.

Serrato-Cruz M A, F Díaz-Cedillo, Barajas-Pérez J S (2008) Composición en el aceite esencial en germoplasma de Tagetes filifolia Lag. de la región centro-sur de México. Agrociencia 42:277-285.

Soule J A (1996) Novel annual and perennial Tagetes. In: Progress in New Crops. J Janick (ed). ASHS Press, Arlington, VA. pp:546-551.

Tariq R M, S N H Naqvi, M I Choudhary, A Abbas (2010) Importance and implementation of essential oil of Pakistanian Acorus calamus Linn., as a biopesticide. Pakistan J. Bot. 42:2043-2050.

Turner B L (1996) The Comps of Mexico: A systematic account of the family Asteraceae, Tageteae and Anthemideae. Phytologia Mem. 10:1-93.

Wink M (1988) Plant breeding: importance of plant secondary metabolites for protection agains pathogens and herbivores. Theor. Appl. Genet. 75:225-233. 